\title{
Electrostatic injection kicker for the KEK digital accelerator
}

\author{
Toshikazu Adachi ${ }^{1,2, *}$ and Tadamichi Kawakubo ${ }^{1, \dagger}$ \\ ${ }^{1}$ High Energy Accelerator Research Organization (KEK), Tsukuba, Ibaraki 305-0801, Japan \\ ${ }^{2}$ The Graduate University for Advanced Studies (KEK), Tsukuba, Ibaraki 305-0801, Japan
}

(Received 25 January 2013; published 28 May 2013)

\begin{abstract}
An electrostatic injection kicker (ES-Kicker) has been developed and installed in the KEK digital accelerator, which is a synchrotron aimed at accelerating all ion species. The ES-Kicker kicks an injected ion beam horizontally into the ring orbit and consists of two main electrodes for electric field generation and three intermediate electrodes to correct field homogeneity. In our single-turn injection scheme, the circulating beam and the injected beam both pass through the electrode aperture of the kicker, so the kicker field must be turned off before arrival of the first circulating beam. The ES-Kicker is therefore operated in a pulse mode. This means that the excitation circuit for the ES-Kicker must be carefully designed, since the falling edge of the electric field is strongly affected by parasitic capacitance of this circuit, and any remaining field may disturb the circulating beam. This paper describes performance of the ES-Kicker on the basis of simulations and measurement results.
\end{abstract}

DOI: 10.1103/PhysRevSTAB.16.053501

PACS numbers: $29.20 . \mathrm{dk}$

\section{INTRODUCTION}

The KEK digital accelerator (KEK-DA; Fig. 1) is a synchrotron aimed at accelerating all ion species [1-3]. It is a rapid-cycling synchrotron operated at $10 \mathrm{~Hz}$. An electron cyclotron resonance ion source embedded in a $200 \mathrm{kV}$ high-voltage platform generates an ion beam, which is guided through a low-energy beam transport line and injected into the KEK-DA by an electrostatic injection kicker (ES-Kicker) [1]. Before injection, an Einzel lens chopper chops the beam pulse to adjust its duration to less than the revolution time period in the KEK-DA [4,5]. Since the typical revolution time is of the order of $10 \mu$ s (e.g., $12 \mu \mathrm{s}$ for $\mathrm{He}^{1+}$ of $200 \mathrm{keV}$ ), the injected beam duration is a few microseconds.

An ion beam enters the ES-Kicker installed in section S1 and is kicked by $11.25^{\circ}$ regardless of ion species. Table I lists ES-Kicker parameters.

To obtain good electric field homogeneity in the kicker gap, there are two choices for the structure of the kicker. One is placing two electrodes in parallel, whose shape is calculated to follow a complex curve. The other is placing a number of intermediate electrodes between the two electrodes, whose shapes are all flat.

\footnotetext{
*toshikazu.adachi@kek.jp

†tadamichi.kawakubo@kek.jp
}

Published by the American Physical Society under the terms of the Creative Commons Attribution 3.0 License. Further distribution of this work must maintain attribution to the author(s) and the published article's title, journal citation, and DOI.
The former structure is suitable for making a compact kicker through which a small beam passes [6,7]. However, it takes considerable time to calculate a suitable shape of the electrodes, especially in the case that a high-quality field is needed over a large area. In contrast, the latter structure is easy for obtaining good field homogeneity even over a large area, not only in the horizontal plane but also in the vertical plane. Since a large ion beam is injected into our kicker, we adopt the intermediate electrode structure.

As shown in Fig. 2, the ES-Kicker comprises an anode (the red plane), a ground electrode (the light blue plane), and three intermediate electrodes (the yellow planes). The beam is kicked horizontally by an electric field into the ring orbit when voltage is applied to the anode. The intermediate electrodes are equally spaced between the anode and the ground electrode. To obtain the desired field homogeneity, voltage is equally divided among these intermediate electrodes by resistors.

Since a single-turn injection scheme is adopted, the circulating beam and the injected beam pass through the ES-Kicker, as shown in Fig. 2. The kicker field must be turned off before arrival of the first circulating beam to avoid disturbance. This must occur within a few microseconds in our case, so the ES-Kicker is operated in a pulse mode.

Figure 3 shows the ES-Kicker excitation circuit. A coaxial cable is charged to the required voltage by a resonant charging power supply and discharged just after beam injection to reset the kicker voltage to zero. The next cable charging cycle begins after beam extraction. Figure 4 shows the ES-Kicker operation timing chart, which is characterized by a slow charge and fast discharge. 


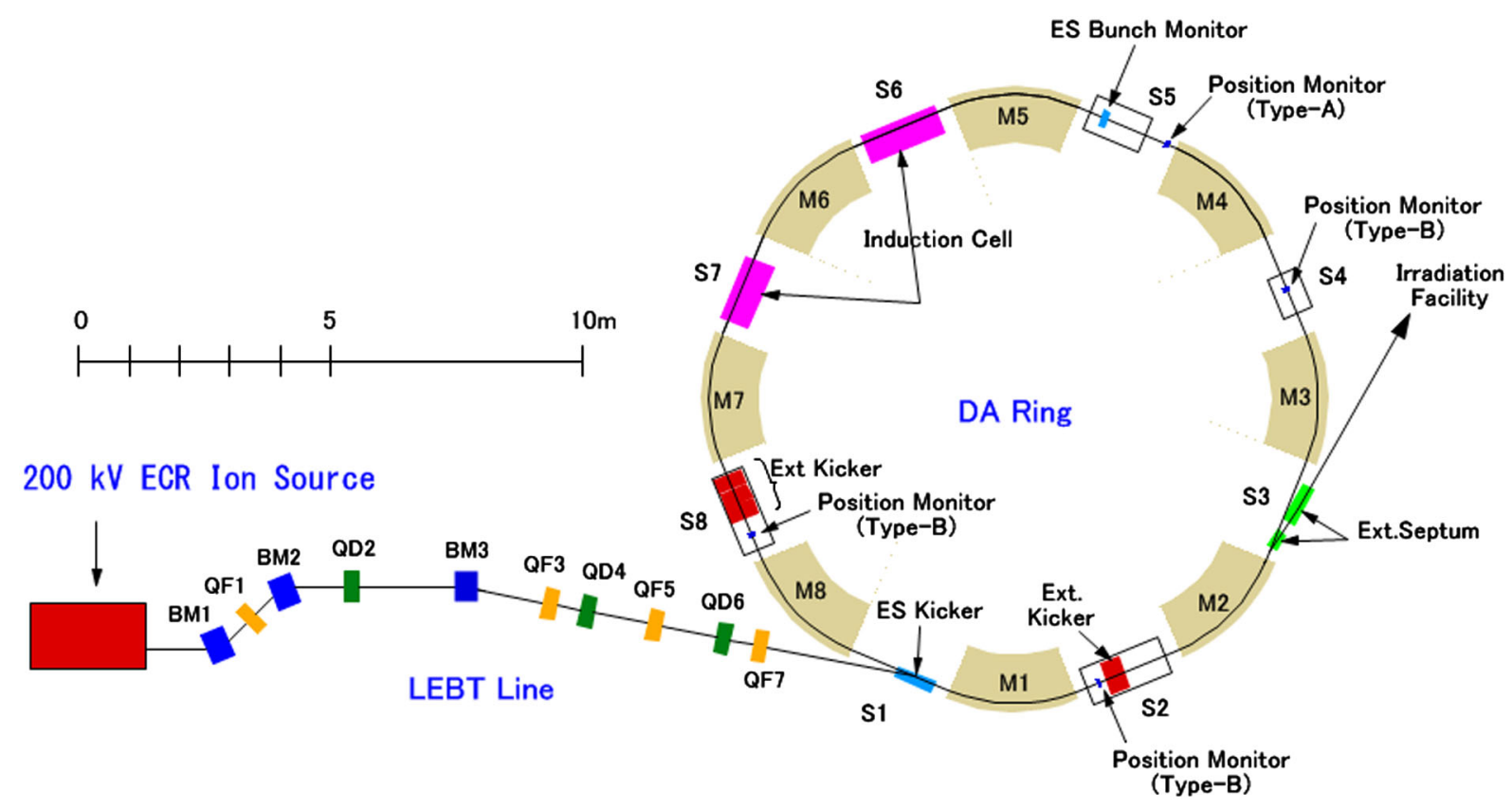

FIG. 1. Schematic view of the KEK-DA.

The cable discharge switching element is a thyratron connected to a matching resistor. Although part of the discharge power is dissipated in the matching resistor, some fraction of the voltage is reflected by a small impedance mismatch between the cable and the matching resistor, causing ringing in the kicker voltage profile.

There remain some issues to be solved for ES-Kicker pulse mode operation, including (1) field homogeneity, (2) residual fields beyond the discharge timing, and (3) effects of the kicker field on the beam quality, such as emittance and momentum spread.

Section II discusses problems related to field homogeneity in the ES-Kicker. Since the injected beam has a large emittance, a wide aperture with good field homogeneity is required. Field distributions have been evaluated for three configurations of the kicker electrodes using the field simulation software TOSCA. Results indicate that three intermediate electrodes should

TABLE I. Parameters of the ES-Kicker.

Kick angle

Electrode length

Aperture (width $\times$ height)

Good field aperture (width $\times$ height)

Maximum repetition rate

Injection beam period

Maximum anode voltage

Maximum charging time

Maximum discharging period be inserted between the anode and ground electrode (Fig. 2).

To confirm field homogeneity in the ES-Kicker, voltage was measured at each electrode by applying AC voltage to the kicker's anode plate. We found that voltage distribution among the electrodes depends on the frequency of the applied AC voltage. Section III presents and discusses these results and configuration of the ES-Kicker.

Section IV discusses pulse response of the ES-Kicker. We use measurement results and simulations to investigate pulse shape of the kicker voltage at each electrode in pulse operation mode (Fig. 4). Behavior of the falling edge of the kicker voltage is particularly affected by

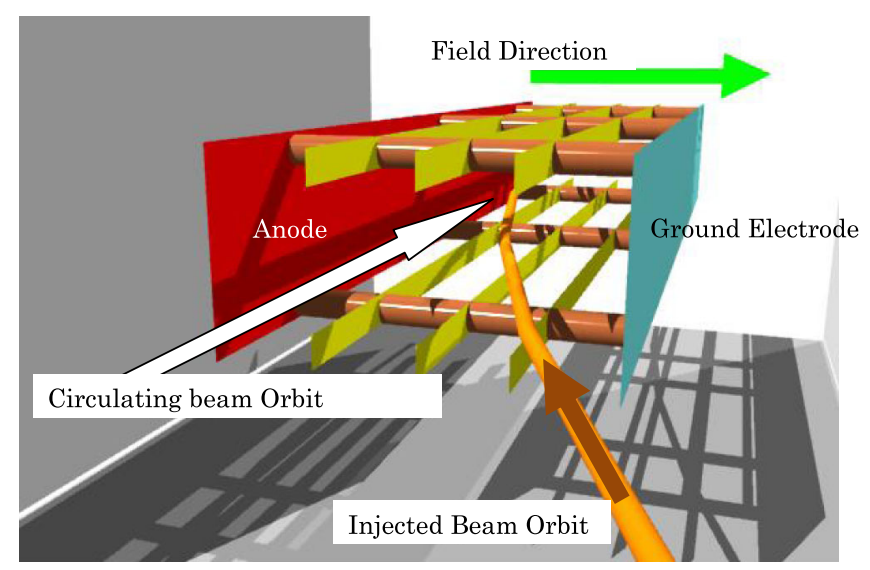

FIG. 2. Schematic view of the ES-Kicker. 


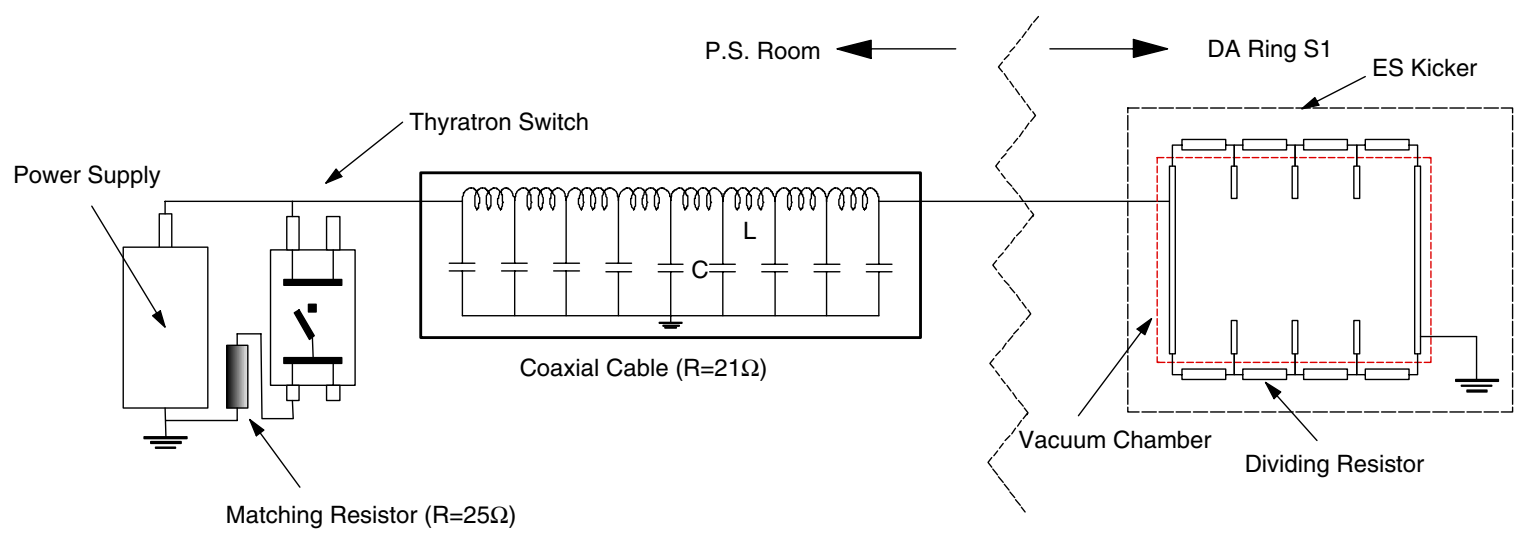

FIG. 3. Schematic of the excitation circuit of the ES-Kicker.

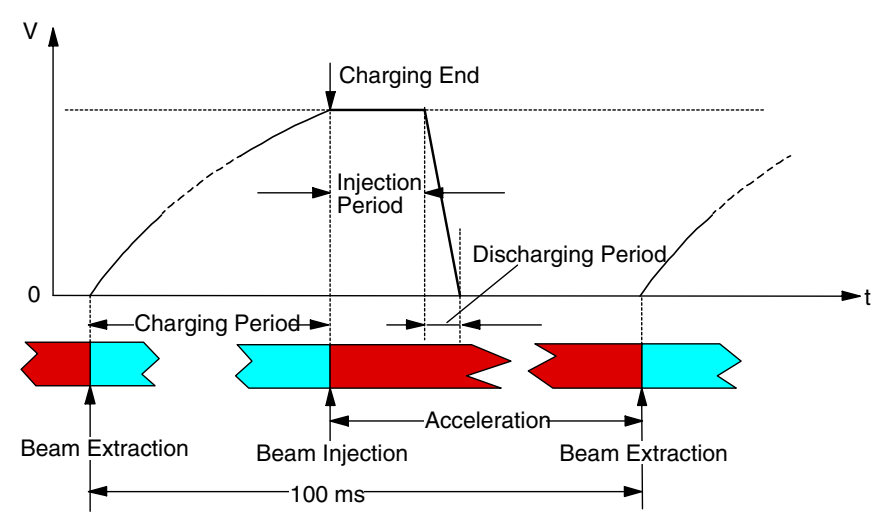

FIG. 4. Timing chart of ES-Kicker operation. parasitic capacitance. Section V discusses beam behaviors affected by the electric field of the ES-Kicker in a simulation that solves the motion equation under the kicker field by the Runge-Kutta method. The simulation result implies that field homogeneity ensured by the intermediate electrodes is effective at preventing emittance blowup. Finally, this work is summarized in the concluding section.

\section{FIELD EVALUATION BY TOSCA}

Field distribution in the ES-Kicker was evaluated by TOSCA, which treats static problems: in this case the problem is electrostatic. Figure 5(a) shows the TOSCA model of

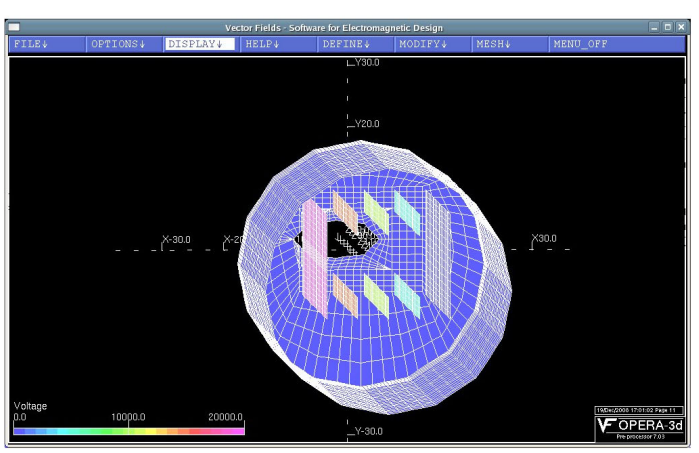

(a)

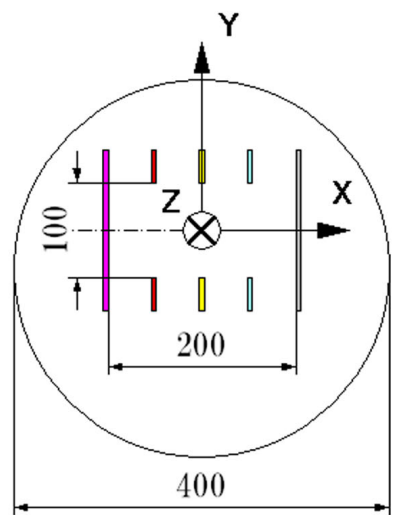

(b)

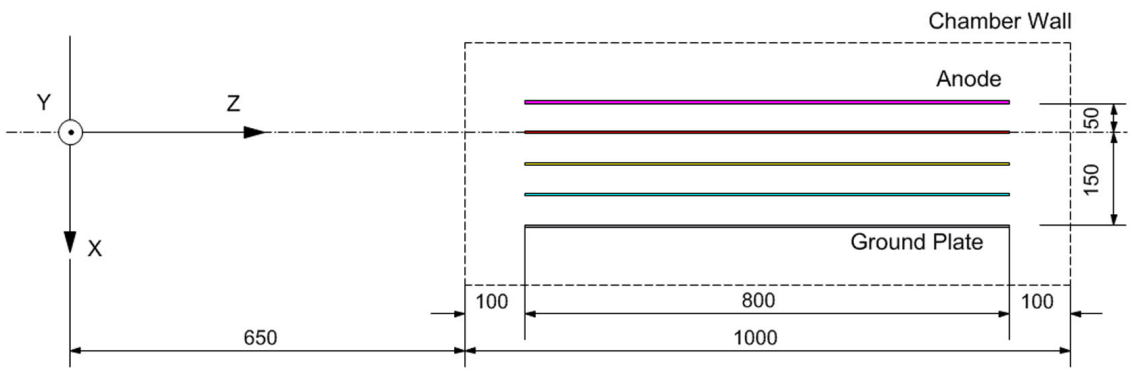

(c)

FIG. 5. (a) TOSCA model of the ES-Kicker. (b) Front view of the ES-Kicker (unit:mm). (c) Top view of the ES-Kicker (unit:mm). 


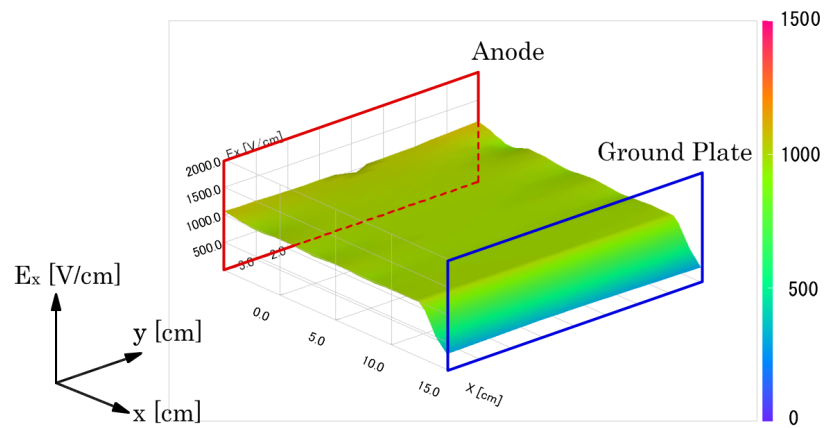

(a)

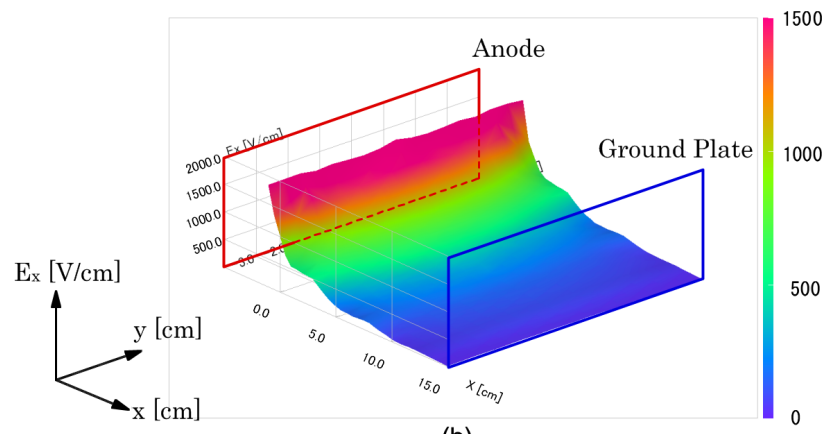

(b)

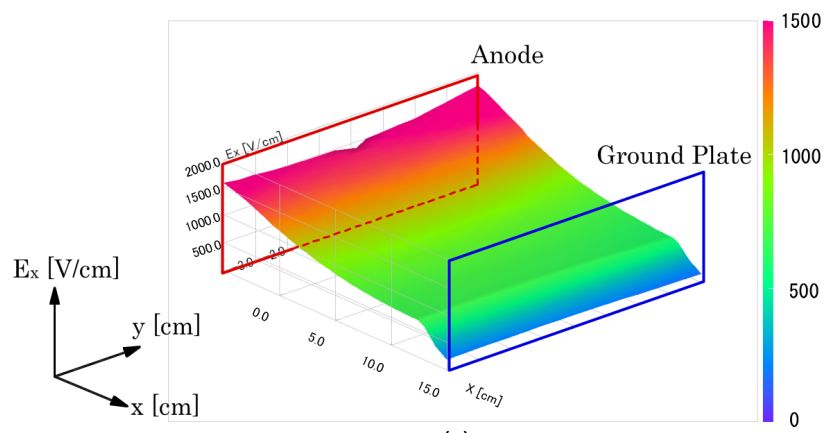

(c)

FIG. 6. (a) $E_{x}$ distribution on the kicker central plane at $z=$ $1150 \mathrm{~mm}$ for Model 0. (b) $E_{x}$ distribution on the kicker central plane at $z=1150 \mathrm{~mm}$ for Model 1. (c) $E_{x}$ distribution on the kicker central plane at $z=1150 \mathrm{~mm}$ for Model 2 .

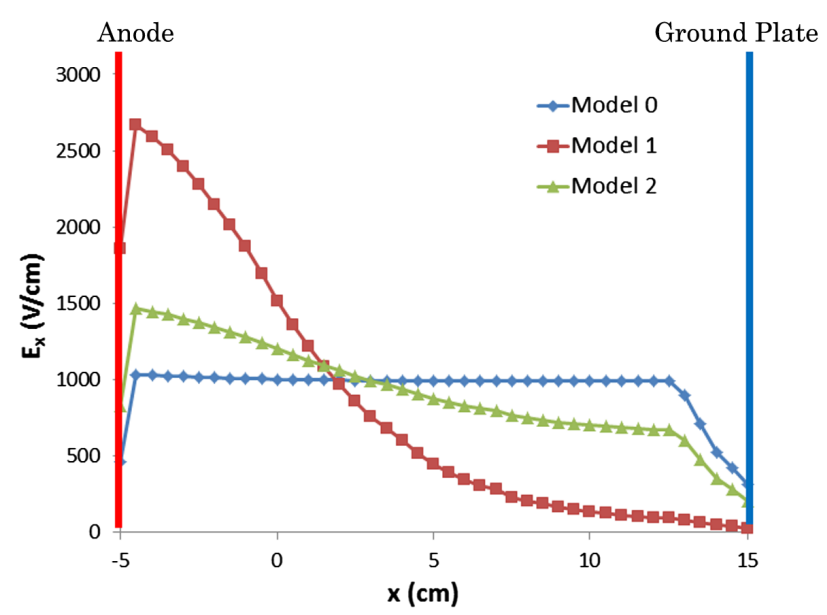

FIG. 7. $x$ dependence of the $E_{x}$ distributions on the kicker central plane at $z=1150 \mathrm{~mm}$ and $y=0 \mathrm{~mm}$ for three models. the ES-Kicker. The kicker is installed in a cylindrical vacuum chamber. Here, a front wall of the chamber is omitted to show the inside view. The anode voltage is set to $20 \mathrm{kV}$. Voltages of intermediate electrodes are $15 \mathrm{kV}$ at the red plane, $10 \mathrm{kV}$ at the yellow plane, and $5 \mathrm{kV}$ at the light blue plane. The voltage at the ground electrode and the chamber wall is $0 \mathrm{~V}$. The diameter of the cylindrical chamber is $400 \mathrm{~mm}$. The $x, y$, and $z$ axes denote the horizontal, vertical, and beam direction, respectively. The kicker region ranges from $z=750$ to $1550 \mathrm{~mm}$, while the chamber ranges from $z=650$ to $1650 \mathrm{~mm}$

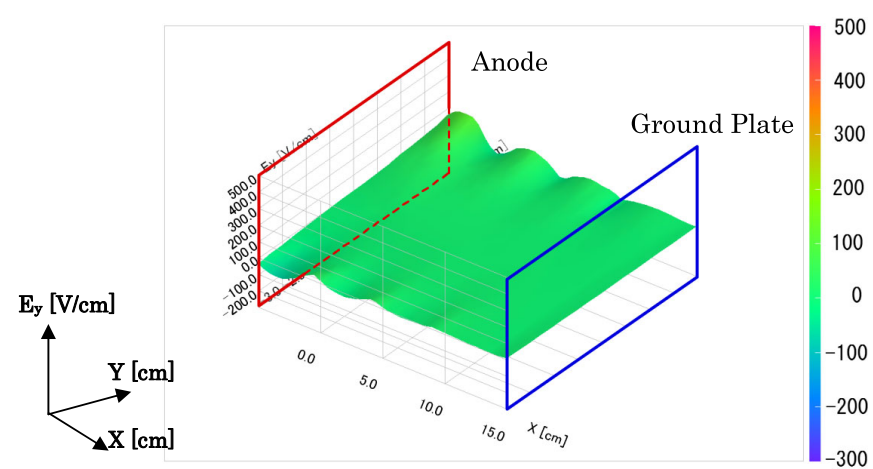

(a)

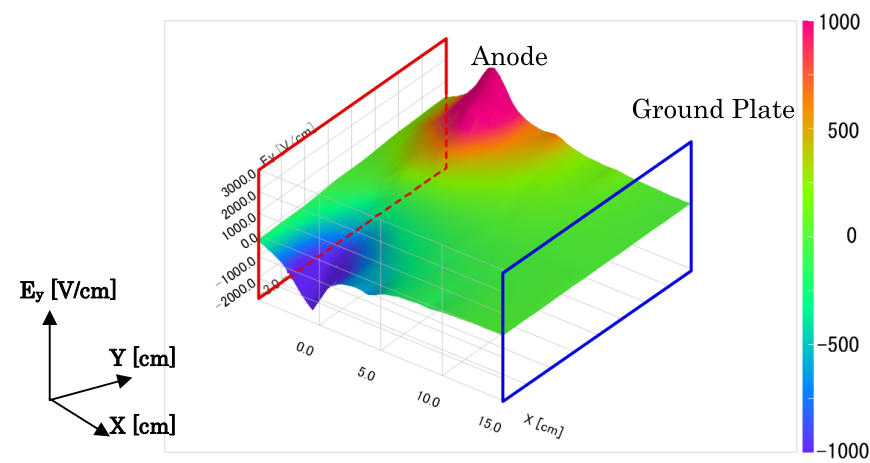

(b)

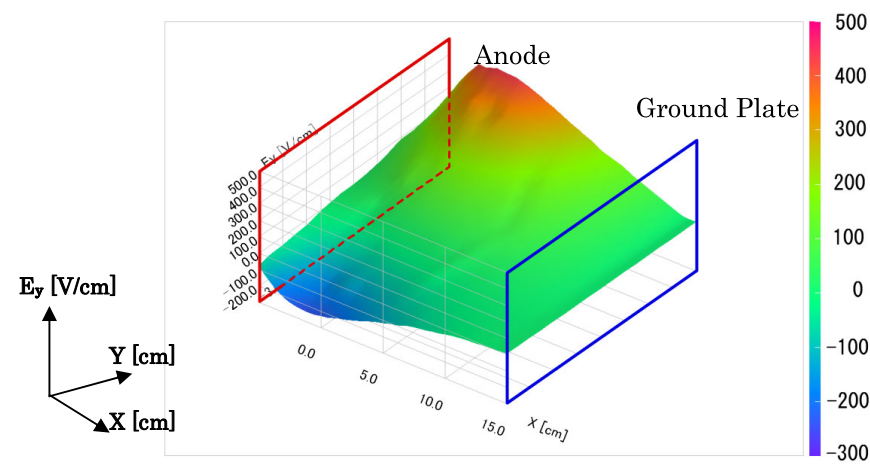

(c)

FIG. 8. (a) $E_{y}$ distribution on the kicker central plane at $z=1150 \mathrm{~mm}$ for Model 0 . (b) $E_{y}$ distribution on the kicker central plane at $z=1150 \mathrm{~mm}$ for Model 1. (c) $E_{y}$ distribution on the kicker central plane at $z=1150 \mathrm{~mm}$ for Model 2. 


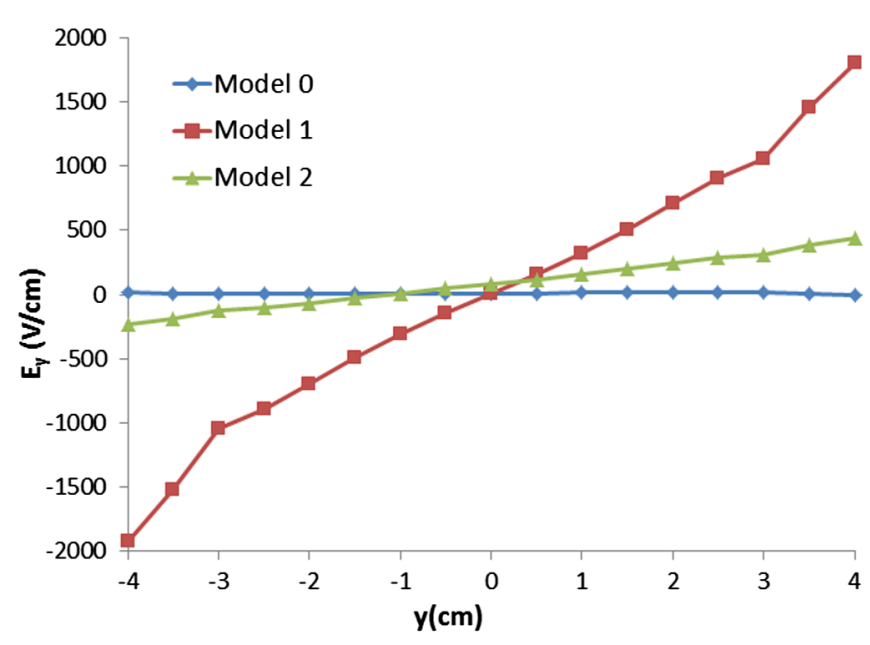

FIG. 9. $y$ dependence of the $E_{y}$ distributions on the kicker central plane at $z=1150 \mathrm{~mm}$ and $x=0 \mathrm{~mm}$ for three models.

[Figs. 5(b) and 5(c)]. Note that the left-handed system is used in these figures.

To investigate the effect of the intermediate electrodes on the field distribution, two different cases are considered, one where the intermediate electrodes are floating but the electric field is required to be normal to the electrodes, the other where these electrodes are removed. The former model is named "Model 1"; the latter, "Model 2"; and the original, "Model 0".

Figures 6(a)-6(c) respectively show $x-y$ dependence of the field component $E_{x}$ for Model 0, Model 1, and Model 2 at $z=1150 \mathrm{~mm}$, the central position along the kicker's beam line. In these figures, extent of the $x-y$ (horizontal) plane is almost equivalent to the kicker transverse aperture, and full scales in the vertical direction are the same, $2000 \mathrm{~V} / \mathrm{cm}$. For Model 0, the field distribution is rather flat, while the field steeply rises near the anode in Model 1. For Model 2, the field slowly decreases along the $x$ axis. For a more quantitative comparison, Fig. 7 shows $E_{x}$ distributions for the three models along the $x$ axis in the median plane $(y=0 \mathrm{~mm})$. Although all plates are symmetric in the $x$ and $y$ directions along the $x=50 \mathrm{~mm}$ and $y=0 \mathrm{~mm}$ lines, the $E_{x}$ distribution is asymmetric. This is caused by the asymmetric potential distribution where the anode is set at $20 \mathrm{kV}$ and the ground plate is connected to the surrounding chamber.

These results imply that a homogeneous kick on the beam in Model 0 is expected due to good homogeneity of the deflecting field. Model 1 also suggests that homogeneity is broken if voltages at the intermediate electrodes are not given definitely.

Figures 8(a)-8(c) respectively show $x-y$ dependence of the field component $E_{y}$ for Model 0, Model 1, and Model 2 at $z=1150 \mathrm{~mm}$. In these figures, the extent of the $x-y$ (horizontal) plane is almost equivalent to the kicker aperture, but their vertical scales are different; the maximum scale is $500 \mathrm{~V} / \mathrm{cm}$ in Model 0 and Model 2, and $3000 \mathrm{~V} / \mathrm{cm}$ in Model 1. In Model 0, the field distribution is almost flat. Small undulations in the fringe region are seen around the intermediate electrodes. For Model 1 and Model 2, the field strength increases with proximity to the anode. The field around the anode becomes particularly large in Model 1. Since the field direction is opposite with respect to $y=0$, some blowup of the vertical emittance may be caused by such a field distribution. For a more quantitative comparison, Fig. 9 shows the $E_{y}$ distributions for the three models along the $y$ axis at $x=0 \mathrm{~mm}$ and $z=1150 \mathrm{~mm}$.

As mentioned above, the intermediate electrodes, whose voltages are fixed at definite values, improve the field homogeneity (Model 0). If they are floating, however, field

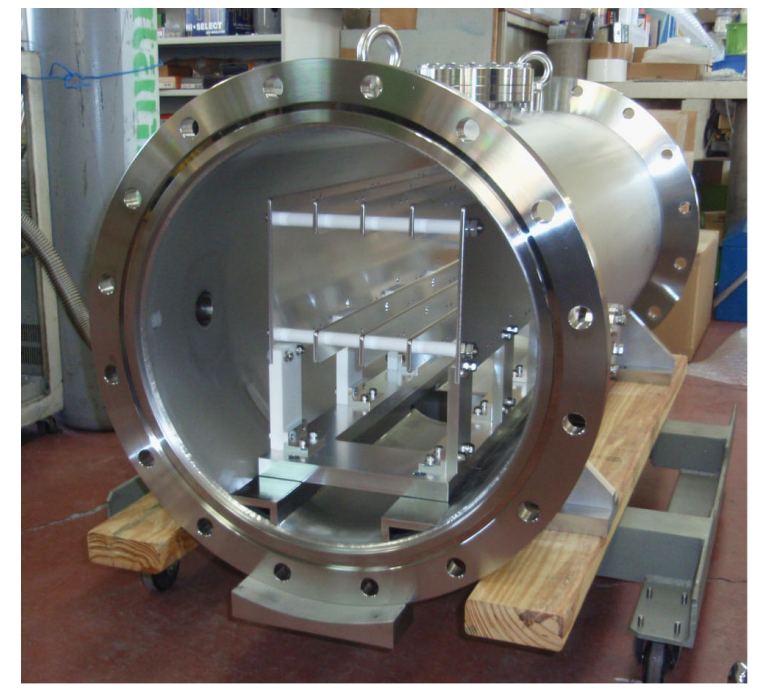

(a)

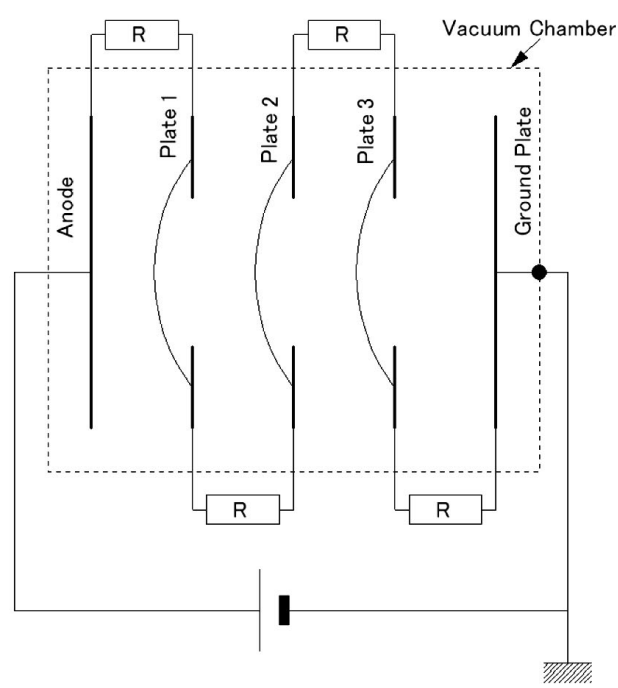

(b)

FIG. 10. (a) Photograph of the ES-Kicker. (b) Schematic view of a dividing resistor configuration. 
homogeneity becomes worse than the case of Model 2. The voltage of each electrode must therefore be fixed at an appropriate value to ensure field homogeneity. Dividing resistors are thus connected between the anode and intermediate electrodes. This method is quite useful for DC fields, but not necessarily for AC fields that may be disturbed due to parasitic capacitance. The dividing impedance of the $i$ th electrode $Z_{i}$ in the AC mode is given by the following formula:

$$
Z_{i}=\frac{R}{1+j \omega R C_{i}}
$$

Here, $R$ is the dividing resistor, $C_{i}$ is the parasitic capacitance of the $i$ th electrode, and $\omega$ is the angular frequency of the applied AC voltage. If $\omega R C_{i} \ll 1$, parasitic capacitance can be ignored and homogeneity is ensured even for AC operation. Note that parasitic capacitance can be controlled by introducing additional capacitors.

\section{VOLTAGE MEASUREMENT AT THE KICKER ELECTRODES}

Figure 10(a) shows a photograph of the ES-Kicker installed in a vacuum chamber without wiring. To fix a definite voltage, each electrode is connected to dividing resistors set outside of the vacuum to eliminate outgassing from the resistors [Fig. 10(b)].

The voltage at each electrode is measured by applying AC voltages with frequencies of $1,10,100$, and $1 \mathrm{MHz}$ between the anode and ground electrode. Measurements were performed for three resistor configurations: no resistors, $R=1 \mathrm{M} \Omega$, and $R=500 \Omega$. Figure 11 shows the results. Here, voltages are normalized by the anode voltage, and the $x$ position is normalized by the lengths from the anode to the ground electrode, that is, $1.0,0.75,0.5$, and 0.25 from the anode to plate 3 .

In the case of no resistors [Fig. 11(a)], the voltage distribution along the electrodes (the anode, plate 1, plate 2 , and plate 3 ), which may be determined by the floating capacitance, is nonlinear and independent of frequency. In the case of $R=1 \mathrm{M} \Omega$ [Fig. 11(b)], however, the voltage distribution changes drastically at $10 \mathrm{kHz}$. At frequencies above $10 \mathrm{kHz}$, we obtain a voltage distribution similar to that in the case of no resistor, since the contribution of parasitic capacitance dominates the resistor.

On the other hand, in the case of $R=500 \Omega$ [Fig. 11(c)], the measured voltage distribution is reasonably linear. This implies that the capacitive impedance, given by $1 / \omega C$, is much lower than $1 \mathrm{M} \Omega$ but much larger than $500 \Omega$ under AC operation above $1 \mathrm{kHz}$. To ensure linear voltage distribution in the horizontal direction along the electrodes, it is therefore desirable to reduce resistance as much as possible. Attaining low resistance is difficult, however, because a

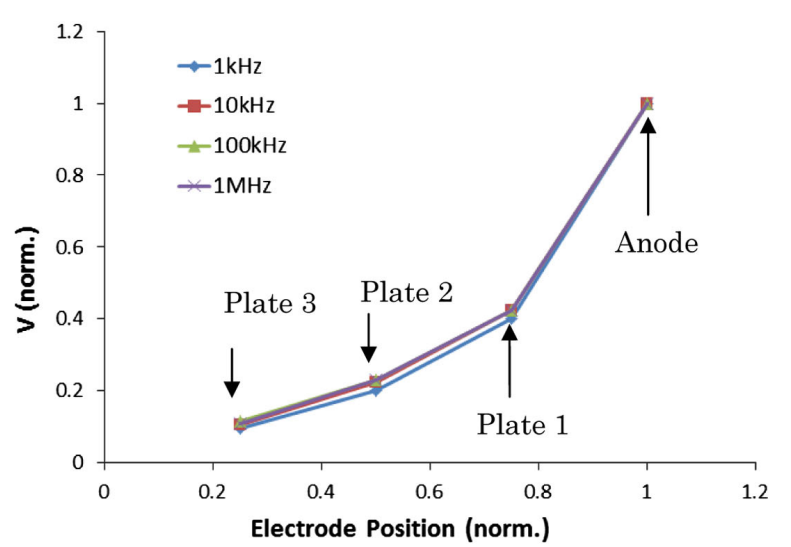

(a)

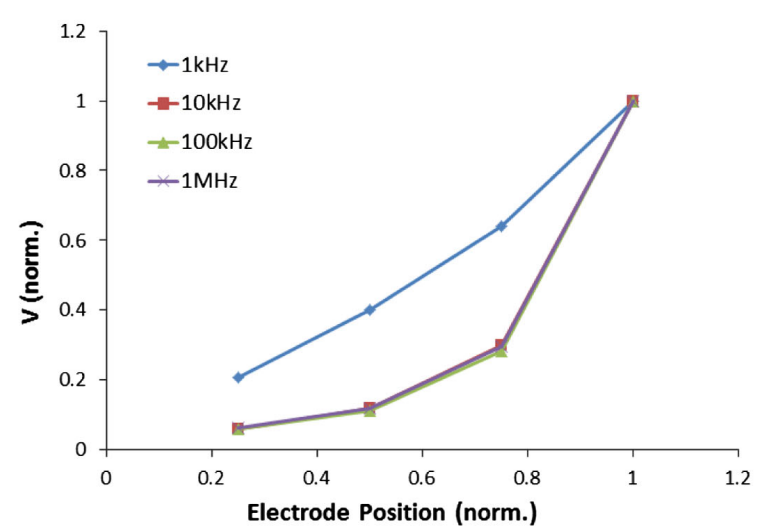

(b)

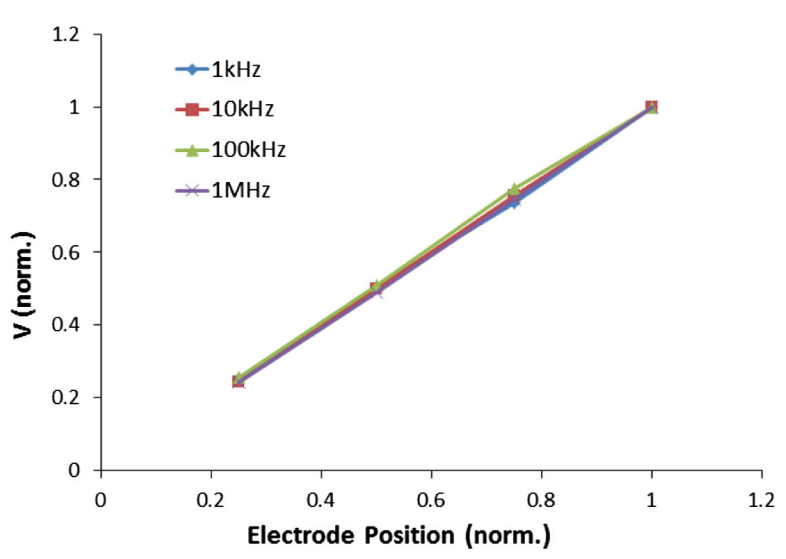

(c)

FIG. 11. (a) Measured voltage distribution in the case of no resistors. (b) Measured voltage distribution in the case of $R=1 \mathrm{M} \Omega$. (c) Measured voltage distribution in the case of $R=500 \Omega$.

large current is supplied to maintain the required anode voltage.

\section{PULSE OPERATION OF THE ES-KICKER}

The ES-Kicker is operated using the circuit shown in Fig. 3. Here, a $1 \mathrm{M} \Omega$ dividing resistor is selected. Since the maximum available charging time is $3 \mathrm{~ms}$, a typical 


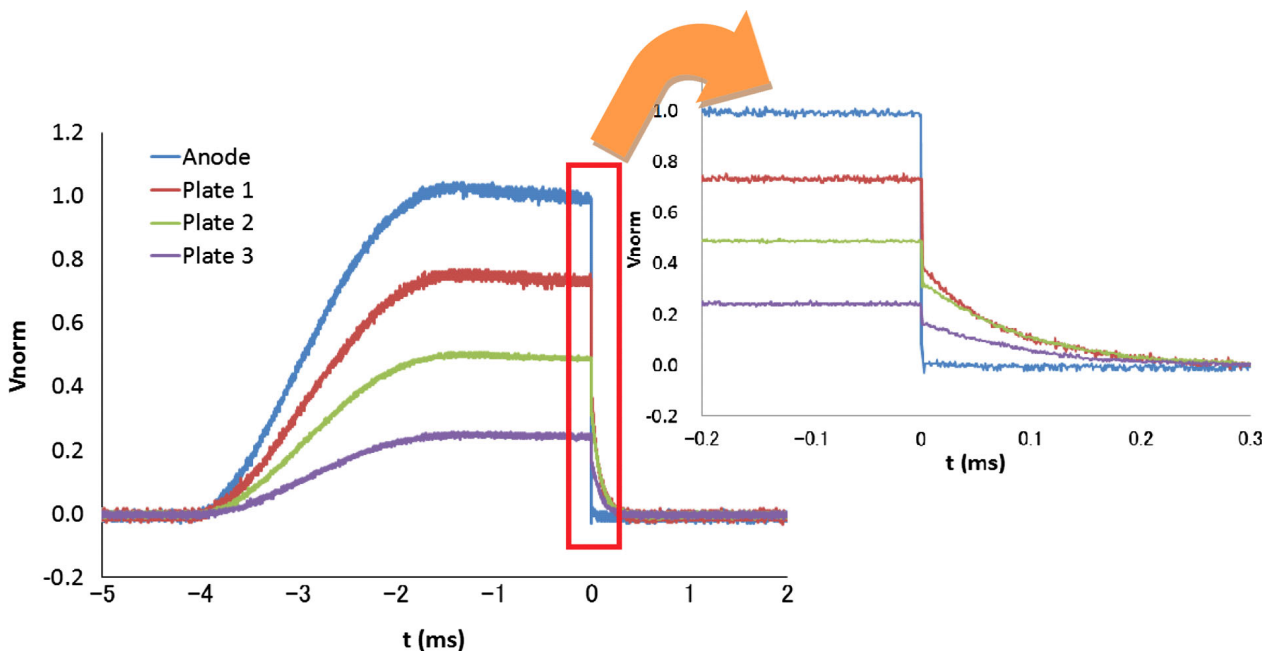

FIG. 12. Voltage waveforms measured at the anode and the intermediate electrodes, and zoomed-up waveforms around the falling edge of the voltage pulse are also shown in the upper right.

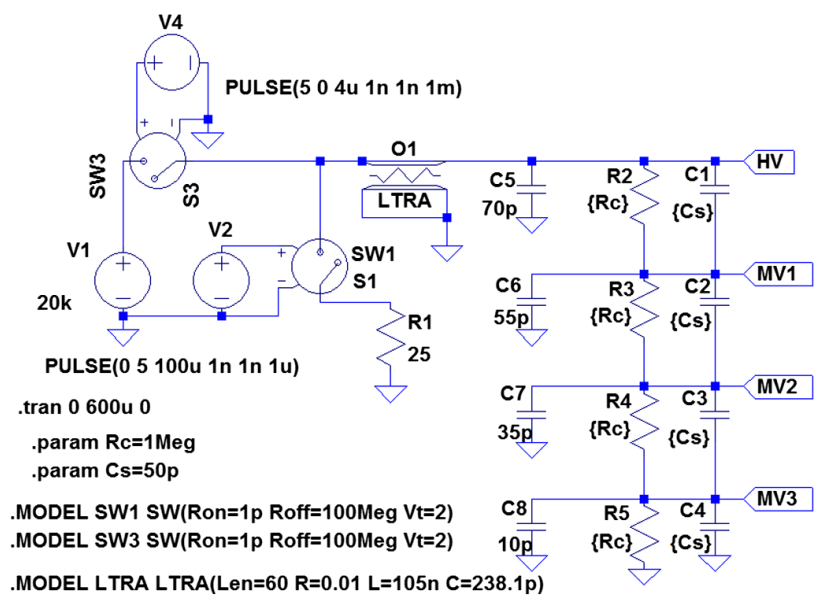

(a)

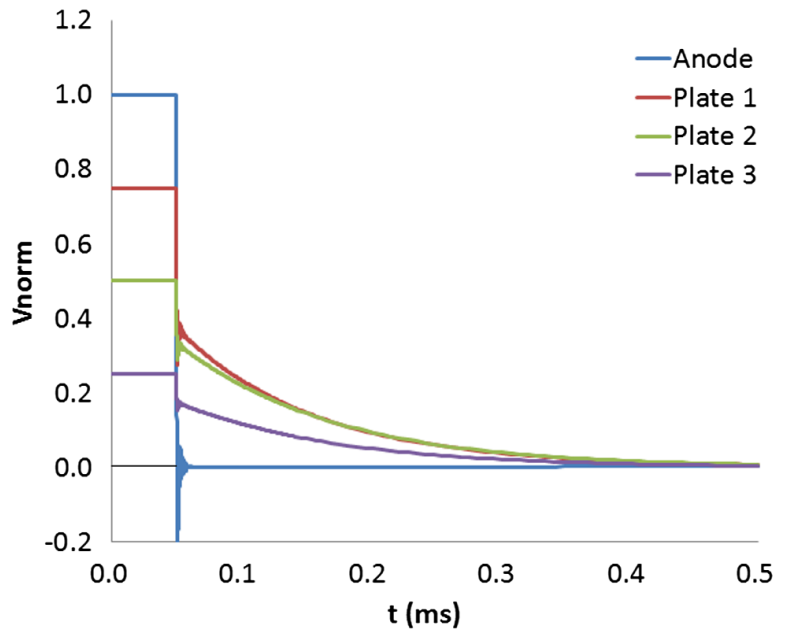

(b)

FIG. 13. (a) Equivalent circuit of the ES-Kicker system giving the long tail at the falling edge. (b) Simulation result of the voltage pulse around the falling edge. Here, the voltage is normalized by the anode voltage at the flattop.

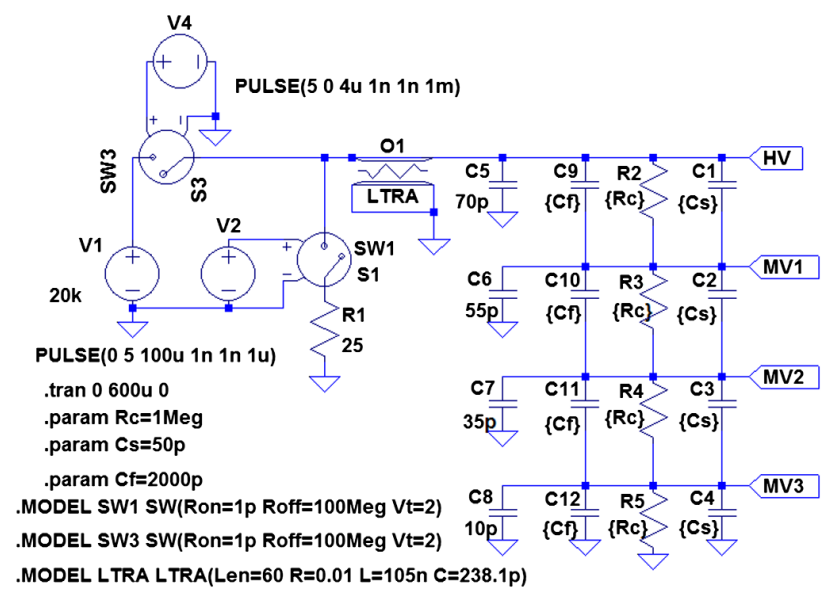

(a)

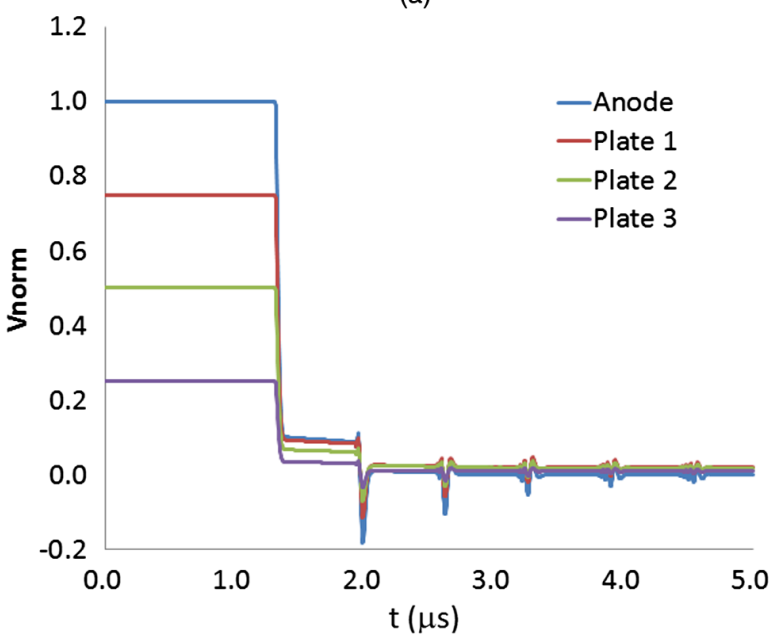

(b)

FIG. 14. (a) Modified equivalent circuit of the ES-Kicker system. (b) Simulation result of the voltage pulse around falling edge after optimization of capacitances C9-C12. Here, the voltage is normalized by the anode voltage at the flattop. 


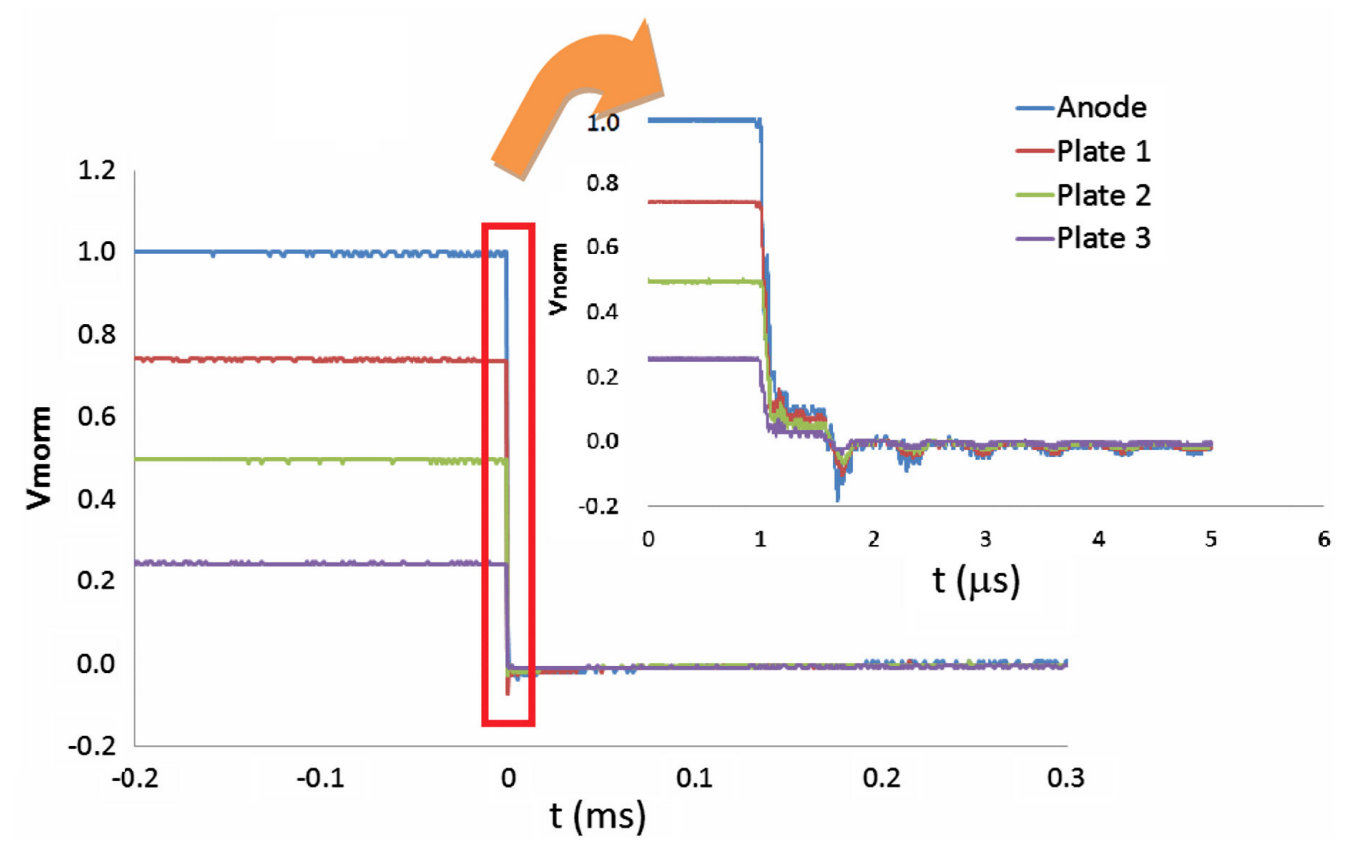

FIG. 15. Voltage waveforms measured at the anode and the intermediate electrodes around the turn-off timing. Here, the voltage is normalized by the anode voltage at the flattop. Expanded waveforms around the falling edge of the voltage pulse are also shown in the upper right.

frequency is about $300 \mathrm{~Hz}$. Therefore, $1 / \omega C$ still exceeds $1 \mathrm{M} \Omega$, and field homogeneity can be expected. Figure 12 shows voltage waveforms measured at the anode and the intermediate electrodes. Here, voltages at the flattop successively decrease by steps of $1 / 4$ the anode voltage from the anode to the last intermediate electrode (Plate 3). The anode voltage at the flattop normalizes the voltage. Although the desired voltage distribution among the electrodes is obtained at the flattop, voltage pulses at the three intermediate electrodes have long tails of approximately $200 \mu \mathrm{s}$, and each tail behaves differently. These long tails disturb the circulating beam, since a typical revolution time is $12 \mu \mathrm{s}$ for low-charge ions of $200 \mathrm{keV}$.

The characteristic frequency around the falling edge exceeds a few tens of kilohertz. Capacitive impedance should therefore contribute to the falling behavior of the voltage pulse. To investigate the contribution of parasitic capacitance, an equivalent circuit representing the ESKicker system [Fig. 13(a)] was studied using the circuit simulation software LT-SPICE.

First, the parasitic capacitance of each electrode is optimized to reproduce the long tail at the falling edge. Figure 13(a) shows the resulting equivalent circuit, where HV, MV1, MV2, and MV3 are voltages at the anode and the three intermediate electrodes, respectively. C1, C2, C3, and $\mathrm{C} 4$ are parasitic capacitances between neighboring electrodes, and C5, C6, C7, and C8 are parasitic capacitances between each electrode and the ground. The dividing resistors are R2, R3, R4, and R5. Figure 13(b) shows the simulation results.
Additional capacitances $\mathrm{C} 9, \mathrm{C} 10, \mathrm{C} 11$, and $\mathrm{C} 12$ are added to the dividing resistors in parallel as an obvious method of reducing the long tails. Figure 14(a) shows the resulting modified circuit. Figure 14(b) shows simulation results, where the long tail is reduced in the case of $2000 \mathrm{pF}$ for $\mathrm{C} 9$ through $\mathrm{C} 12$. Although there remain small pulse structures caused by reflection at the matching resistor, we expect that such small residual voltages do not significantly disturb the circulating beam.

Following the simulation results, a $2000 \mathrm{pF}$ capacitance was connected to each $1 \mathrm{M} \Omega$ resistor in parallel. As shown in Fig. 15, the kicker voltage successfully turns off within a few hundred nanoseconds, and experimental results agree well with the simulation. We thus expect the kicker voltage to turn off before the first circulating beam enters the ES-Kicker.

\section{SIMULATION OF BEAM BEHAVIOR IN THE ES-KICKER}

Beam behavior inside the ES-Kicker region was simulated using the fourth-order Runge-Kutta method for two kicker configurations, Model 0 (intermediate electrodes) and Model 2 (no intermediate electrodes). Figure 16 shows the straight section S1 of the DA ring used for beam injection. Dashed lines indicate the simulation region (see also Fig. 1).

A low-charge ion of $A / Q=4$ beam is injected into the simulation region. At the simulation start, the beam center is $\left(23 \mathrm{~mm},-11.25^{\circ}\right)$ in the horizontal phase space and $\left(0.0 \mathrm{~mm}, 0.0^{\circ}\right)$ in the vertical phase space. 


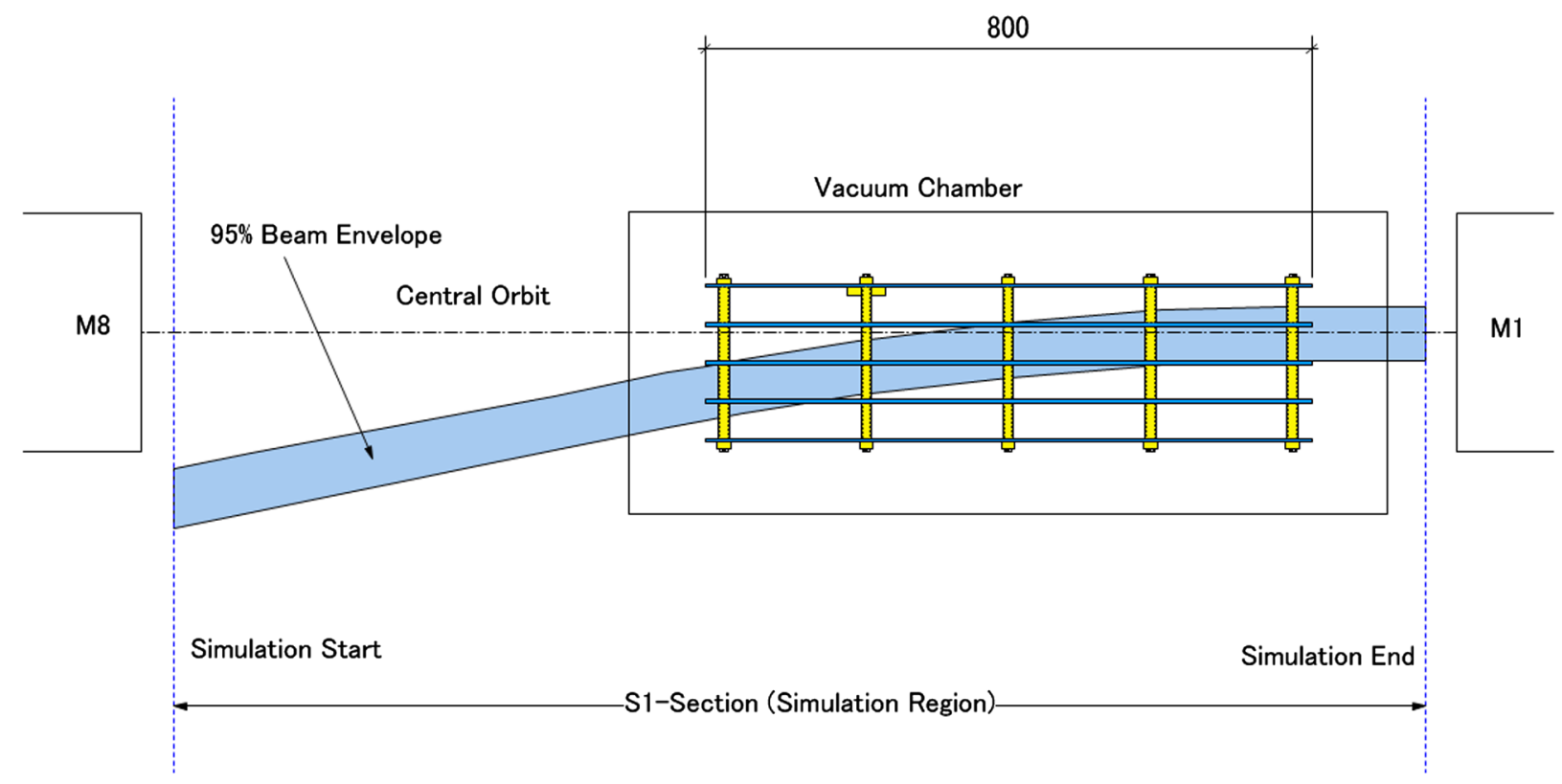

FIG. 16. Top view of the straight section S1 for beam injection including the ES-Kicker. Here, M1 and M8 are ring magnets.

Anode voltage was optimized to $17.7 \mathrm{kV}$ to guide the horizontal beam center to the ring central obit at the end of the simulation region. Table II lists initial beam parameters. Beam emittances are assumed to be the acceptance of the KEK-DA ring.

\section{A. Transverse motion in the ES-Kicker}

To investigate beam excursion in the horizontal phase space, six monitoring positions are set along the beam orbit. At each position, typical horizontal beam motion can be described in the phase space whose origin is the circulating beam orbit in the DA ring [Fig. 17(a)]. If all phase plots on the way to the ring are projected onto the phase space at the exit of the ES-Kicker, we can easily see the beam excursion in the horizontal phase space. Figure 17(b) shows the obtained phase plot for Model 0. Here, the beam propagates from the lower right to the upper left. Figures 18(a) and 18(b) show the initial and final beam distributions in the horizontal and vertical phase spaces, respectively. Red lines indicate Courant-Snyder ellipses with $2 \sigma$ emittance.

Beam emittance was estimated at each monitoring position by tracking 5000 particles. Particle distributions in

TABLE II. Initial values of beam parameters.

\begin{tabular}{lcc}
\hline \hline & Horizontal & Vertical \\
\hline$\beta(\mathrm{m})$ & 3.769 & 2.279 \\
$\alpha$ & 0.309 & 0.687 \\
$2 \sigma$ emittance $(\pi \mathrm{mm}$ mrad $)$ & 165 & 32 \\
\hline \hline
\end{tabular}

phase space are prepared in $z=$ constant planes that correspond to the six monitoring positions. Unfortunately, beam direction is not normal to such a plane, because the beam is injected into the ring with an inclination to the $z$ axis, which is parallel to the circulating beam orbit in the straight section S1. Therefore, the $x-z$ frame should be rotated about the $y$ axis to obtain a $\xi$-s frame for emittance estimation. Figure 19 shows the configuration of the $x-z$ frame and $\xi-s$ frame.

Rotation angle $\theta$ at a monitoring position is given by

$$
\theta=\tan ^{-1}\left\langle\frac{p_{x}}{p_{z}}\right\rangle \equiv \tan ^{-1}\left\langle x^{\prime}\right\rangle
$$

Here, $p_{x}$ and $p_{z}$ are momentum components in the $x-z$ frame and " \langle\rangle " indicates an average value.

Phase space coordinates in the $\xi$-s frame are given by the following formula:

$$
\xi=\frac{(x-\langle x\rangle)}{\sqrt{1+\left\langle x^{\prime}\right\rangle^{2}}}, \quad \xi^{\prime}=\frac{p_{\xi}}{p_{s}}=\frac{x^{\prime}-\left\langle x^{\prime}\right\rangle}{1+x^{\prime}\left\langle x^{\prime}\right\rangle} .
$$

For vertical motion, the direction of motion is different. Therefore, by introducing a new variable, $\eta$, we obtain

$$
\eta \equiv y, \quad \eta^{\prime}=\frac{p_{\eta}}{p_{s}}=\frac{\sqrt{1+\left\langle x^{\prime}\right\rangle^{2}}}{1+x^{\prime}\left\langle x^{\prime}\right\rangle} \frac{p_{y}}{p_{z}} \equiv \frac{\sqrt{1+\left\langle x^{\prime}\right\rangle^{2}}}{1+x^{\prime}\left\langle x^{\prime}\right\rangle} y^{\prime} .
$$

Here, $p_{\xi}$ and $p_{s}$ are momentum components in the $\xi-s$ frame and are given by $p_{x}$ and $p_{y}$ as follows: 


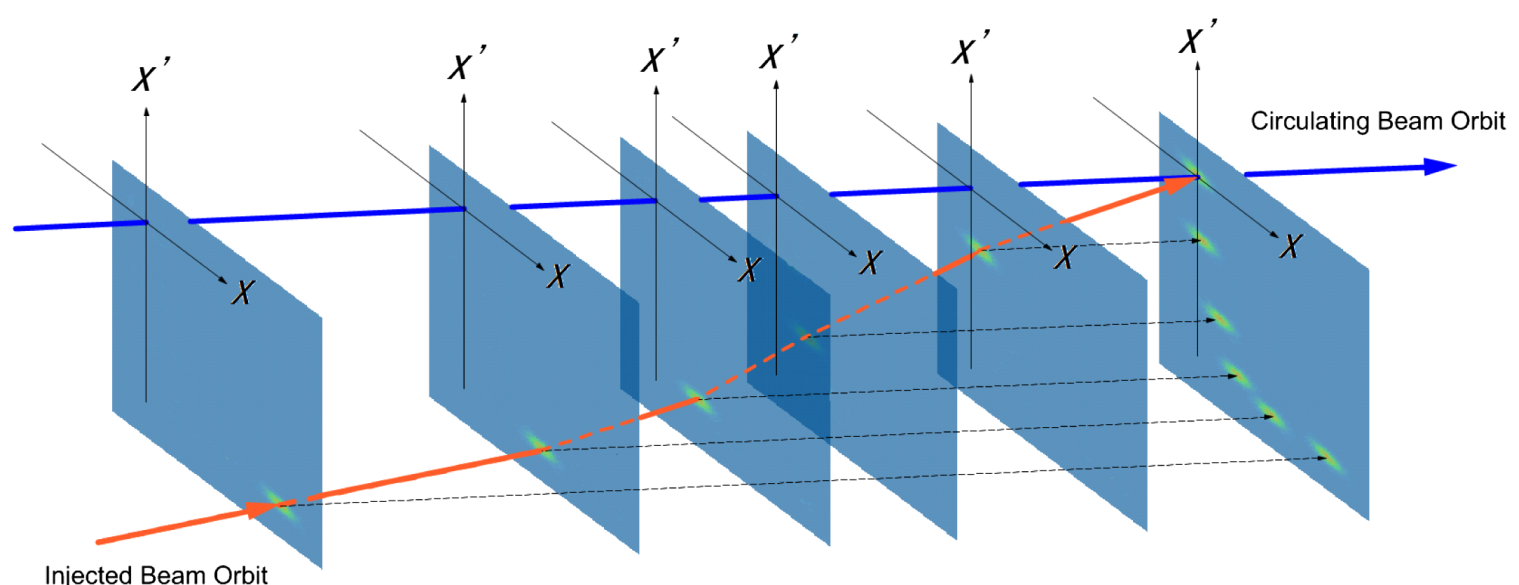

(a)

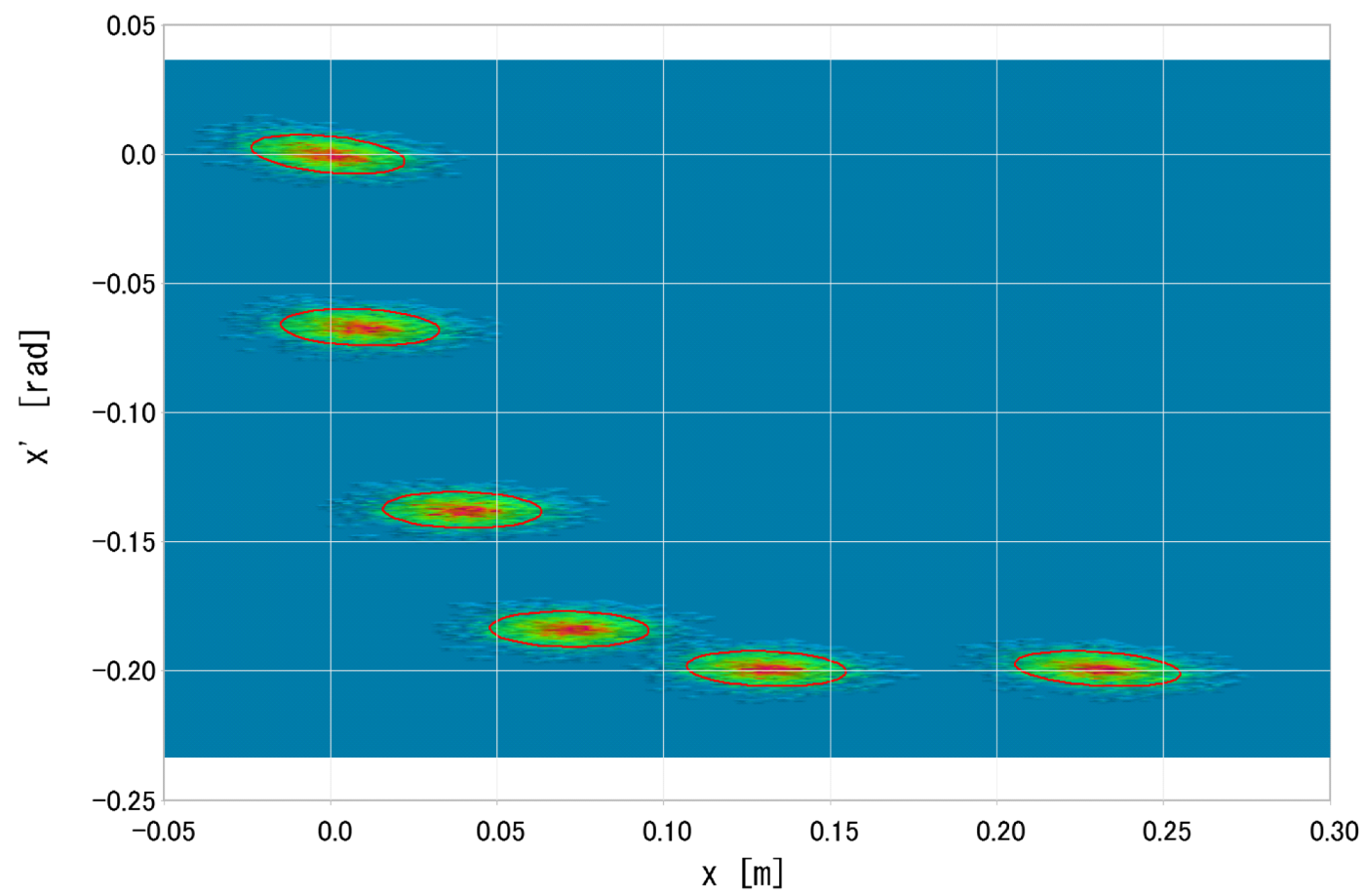

(b)

FIG. 17. (a) Horizontal phase space configuration. (b) Beam excursion in the horizontal phase space, whose origin is the circulating beam orbit, for the Model 0 . The solid red line is the Courant-Snyder ellipse with $2 \sigma$ emittance.

$$
p_{\xi}=p_{x} \cos \theta-p_{z} \sin \theta, \quad p_{s}=p_{x} \sin \theta+p_{z} \cos \theta
$$

The magnitude of emittance $\varepsilon$ was derived from the distribution thus obtained in $\left(\xi, \xi^{\prime}\right)$ or $\left(\eta, \eta^{\prime}\right)$ phase space through a statistical procedure. The explicit forms for horizontal and vertical emittances are given by

$$
\begin{aligned}
& \varepsilon_{H}=\sqrt{\left\langle\xi^{2}\right\rangle\left\langle\xi^{\prime 2}\right\rangle-\left\langle\xi \xi^{\prime}\right\rangle^{2}}, \\
& \varepsilon_{V}=\sqrt{\left\langle\eta^{2}\right\rangle\left\langle\eta^{\prime 2}\right\rangle-\left\langle\eta \eta^{\prime}\right\rangle^{2}} .
\end{aligned}
$$

As the result, the horizontal emittances are $162 \pi \mathrm{mm}$ mrad for the initial beam and $163 \pi \mathrm{mm} \mathrm{mrad}$ for the final beam. The vertical emittances are $32 \pi \mathrm{mm}$ mrad for the initial beam and $35 \pi \mathrm{mm}$ mrad for the final beam. Although a little blowup is seen in both directions, it is an acceptable amount.

Figure 20 shows the simulation results for Model 2. As shown in Fig. 20(a), beam excursion in the horizontal phase space behaves in a slightly different manner from Model 0, which is shown in Fig. 17(b). As the beam approaches the anode, a large phase rotation occurs due to field inhomogeneity (see Fig. 7). 

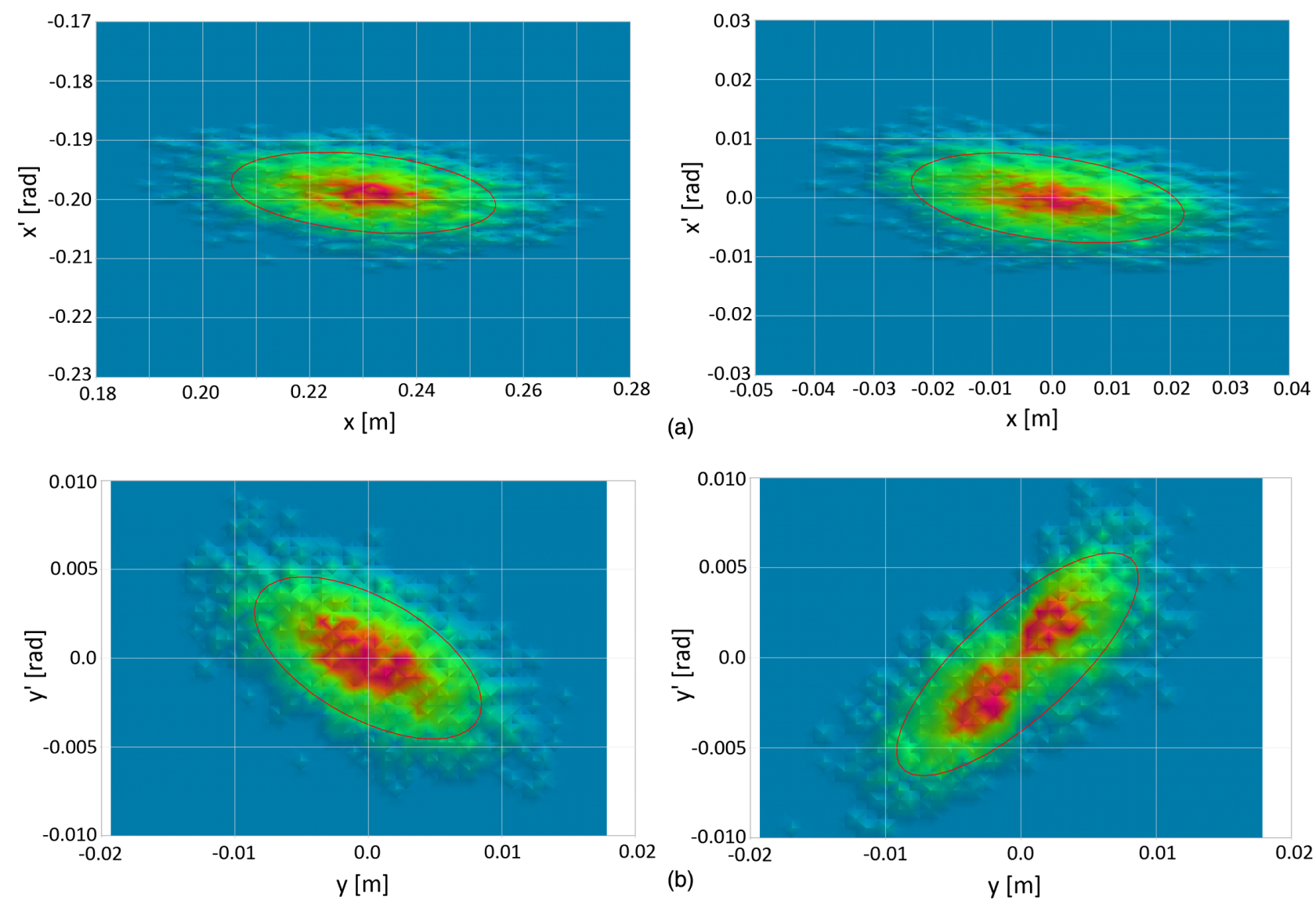

FIG. 18. (a) Beam distribution in the horizontal phase space. Here, the initial and final beam distributions are shown in the left and right figures, respectively. The solid red line is the Courant-Snyder ellipse with $2 \sigma$ emittance. (b) Beam distribution in the vertical phase space. Here, the initial and final beam distributions are shown in the left and right figures, respectively. The solid red line is the Courant-Snyder ellipse with $2 \sigma$ emittance.

Figures 20(b) and 20(c) show the final beam distributions in the horizontal and vertical phase spaces, respectively. The horizontal emittances are $162 \pi \mathrm{mm}$ mrad for the initial beam and $170 \pi \mathrm{mm} \mathrm{mrad}$ for the final beam. Although the emittance become larger than in the case of Model 0, it is still acceptable.

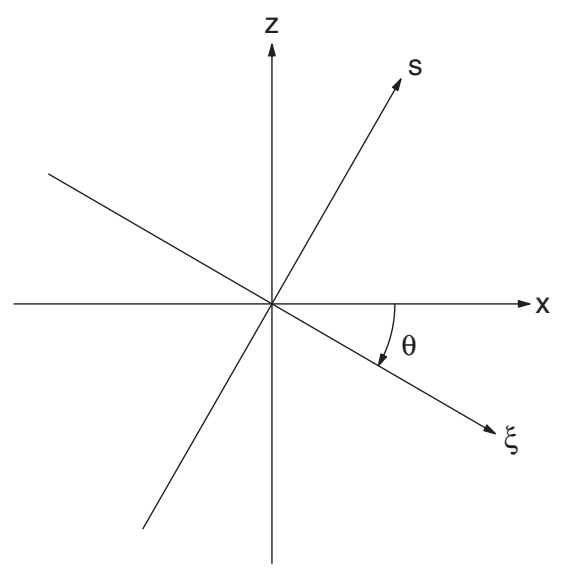

FIG. 19. Configuration of the $x-z$ frame and $\xi-s$ frame.
On the other hand, vertical emittance at the final position is split into two islands by a vertical kick, $10 \mathrm{mrad}$ for the upper island and $-10 \mathrm{mrad}$ for the lower island. Eventually, the effective vertical emittance $(124 \pi \mathrm{mm}$ mrad) becomes 4 times as large as the initial emittance. This is caused by the electric field $E_{y}$, which is negligibly small in the case of Model 0 (see Fig. 9). This type of emittance mismatch cannot be remedied by modifying the injection optics. We therefore conclude that the intermediate electrodes contribute to improvement of the emittance.

\section{B. Momentum change in the ES-Kicker}

Since there is a field component $\left(E_{z}\right)$ along the beam direction in the ES-Kicker, beam momentum after passing through the kicker generally differs from the initial momentum. Figure 21 shows the final momentum distribution when a monochromatic low-charge ion beam of $A / Q=4,200 \mathrm{keV}$, whose momentum is $0.03861 \mathrm{GeV} / c$, is injected into the ES-Kicker. The resulting momentum distributes around an average value of $0.03854 \mathrm{GeV} / c$ with FWHM of $4.83 \times 10^{-6} \mathrm{GeV} / c$. 


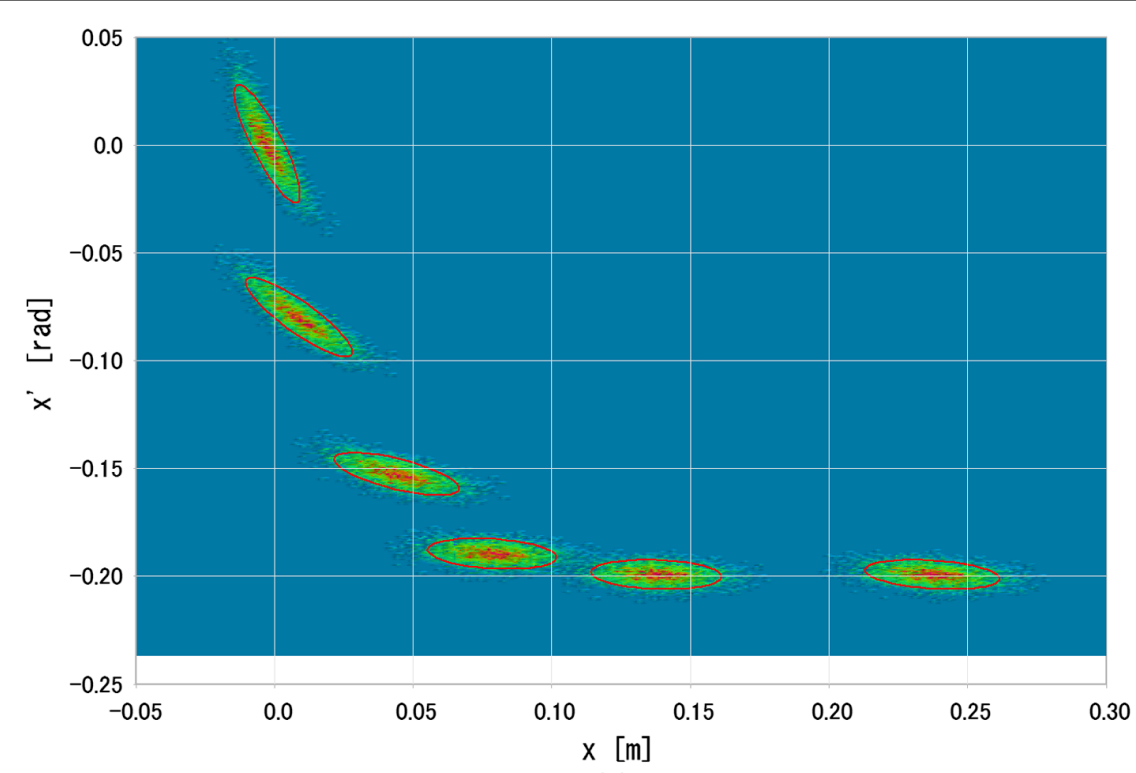

(a)

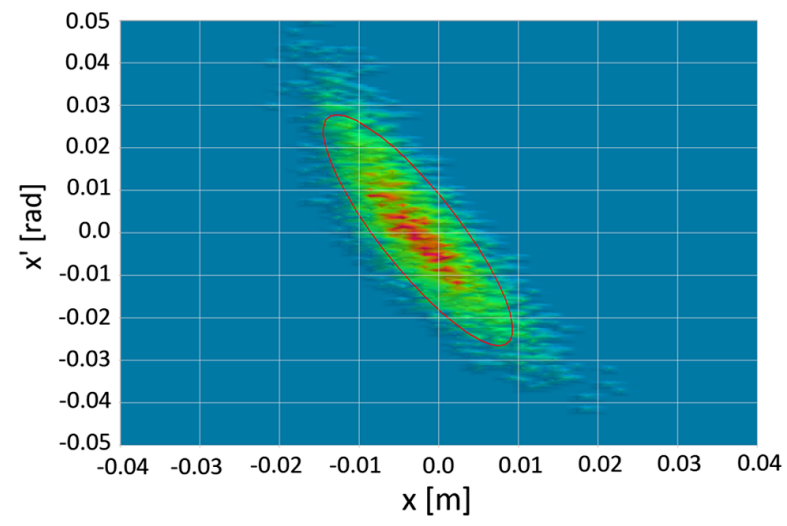

(b)

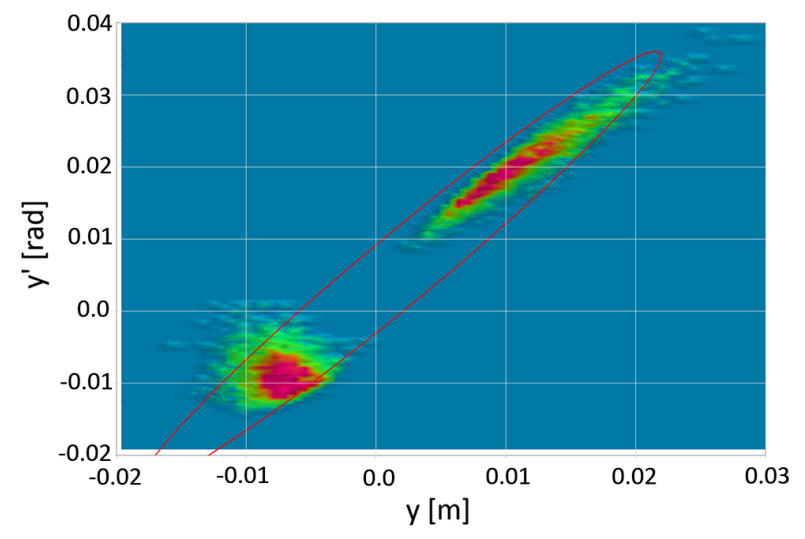

(c)

FIG. 20. (a) Beam excursion in the horizontal phase space for Model 2. The red line is the Courant-Snyder ellipse with $2 \sigma$ emittance. (b) Final beam distribution in the horizontal phase space. (c) Final beam distribution in the vertical phase space.

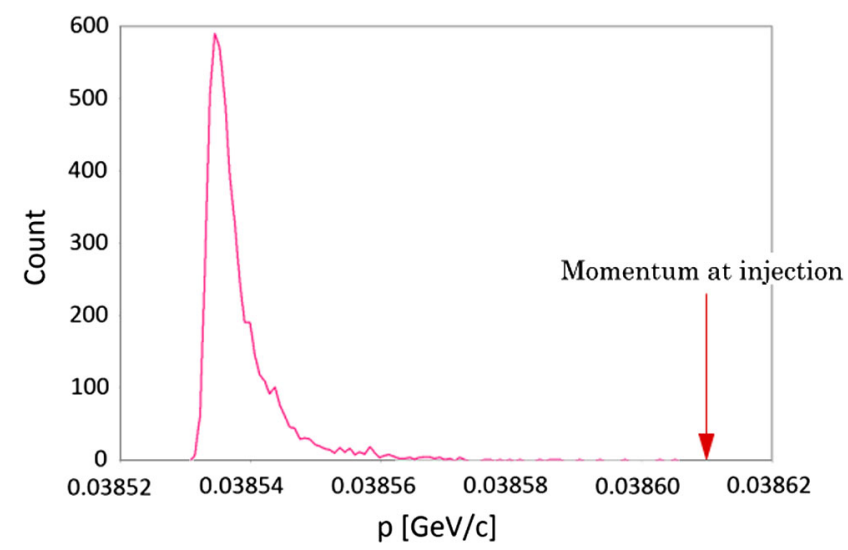

FIG. 21. Final momentum distribution.
Beam momentum is therefore reduced by a fraction of $1.8 \times 10^{-3}$, and the relative momentum spread amounts to $1.3 \times 10^{-4}$ in FWHM.

\section{CONCLUSION}

The ES-Kicker used for beam injection into the DA ring has been designed and its performance investigated. The kicker comprises an anode, a ground electrode, and three intermediate electrodes. The intermediate electrodes ensure field homogeneity inside the kicker aperture. To confirm the contribution of the intermediate electrodes to field homogeneity, field distribution was evaluated using TOSCA for cases with and without 
intermediate electrodes, and the obtained field distributions were used to perform a beam simulation. The results imply that the intermediate electrodes and suitable voltages are needed to avoid fatal beam blowup, especially in the vertical phase space, because this configuration results in good field homogeneity. For such purposes, a dividing resistor is connected to each electrode. Unfortunately, the falling edge of the kicker voltage has a long tail due to parasitic capacitance, and this long tail must be reduced to less than a few microseconds to avoid disturbing the circulating beam. A large capacitance of $2000 \mathrm{pF}$ was connected in parallel to the dividing resistor, drastically reducing the tail length.

Since there is a field component along the beam direction, the beam is accelerated and decelerated in the kicker. Simulation results imply that the monochromatic momentum of the beam at the kicker entrance slightly decreases to the averaged momentum, with some distribution at the exit of the kicker.

The KEK-DA is now being commissioned. The ESKicker operates stably and successfully guides the injected beam into the DA ring.

\section{ACKNOWLEDGMENTS}

The authors acknowledge Mr. T. Yoshii (Nagaoka University of Technology) for his help in circuit simulations, and Professor K. Okamura for his help in reducing the tail at the falling edge of the voltage. We also express our gratitude to Professor K. Takayama and the KEK-DA project members for various discussions of this paper. The present study was financially supported by Grant-In-Aid for Creative Scientific Research (S) (KAKENHI No. 20224005) and (A) (KAKENHI No. 23240082).

[1] K. Takayama, Y. Arakida, T. Iwashita, Y. Shimosaki, T. Dixit, and K. Torikai, J. Appl. Phys. 101, 063304 (2007); 103, 099903(E) (2008); Patent No. 3896420, PCT/JP2006/ 308502, 2006; Induction Accelerators, edited by $\mathrm{K}$. Takayama and R.J. Briggs (Springer-Verlag, BerlinHeidelberg, 2011), Chap. 12.

[2] K. Takayama, Y. Arakida, T. Dixit, T. Iwashita, T. Kono, E. Nakamura, K. Otsuka, Y. Shimosaki, K. Torikai, and M. Wake, Phys. Rev. Lett. 98, 054801-4 (2007).

[3] T. Iwashita, T. Adachi, K. Takayama, K. W. Leo, T. Arai, Y. Arakida, M. Hashimoto, E. Kadokura, M. Kawai, T. Kawakubo, Tomio Kubo, K. Koyama, H. Nakanishi, K. Okazaki, K. Okamura, H. Someya, A. Takagi, A. Tokuchi, and M. Wake, Phys. Rev. ST Accel. Beams 14, 071301 (2011).

[4] T. Adachi, T. Arai, K. W. Leo, K. Takayama, and A. Tokuchi, Rev. Sci. Instrum. 82, 083305 (2011).

[5] K. W. Leo, T. Adachi, T. Arai, and K. Takayama, Phys. Rev. ST Accel. Beams 16, 043502 (2013).

[6] D. Alesini, S. Guiducci, F. Marcellini, and P. Raimondi, in Proceedings of the 10th European Particle Accelerator Conference, Edinburgh, Scotland, 2006 (EPS-AG, Edinburgh, Scotland, 2006).

[7] C. Belver-Aguilar, A. Faus-Golfe, M. J. Barnes, and F. Toral, in Proceedings of the International Beam Instrumentation Conference, IBIC2012, Tsukuba, Japan, 2012 [http:// www.jacow.org/]. 